\title{
CORRECCIONES A LA TAXONOMÍA Y NOMENCLATURA DEL GÉNERO PSYCHOTRIA S. L. EN LAS FLORAS DE CUBA, LA ESPAÑOLA Y JAMAICA
}

\author{
A. L. Borhidi ${ }^{1}$ y R. Oviedo-Prieto ${ }^{2}$ \\ ${ }^{1}$ Instituto de Biología, Universidad de Pécs, H-7624 Pécs, Ifjúság útja 6, Hungría \\ E-mail: borhidi@ttk.pte.hu \\ ${ }^{2}$ Instituto de Ecología y Sistemática, Ministerio de Ciencia \\ Tecnología y Medio Ambiente (CITMA) La Habana, Cuba; E-mail: roviedo@ceniai.inf.cu
}

(Received 1 August, 2015; Accepted 25 August, 2015)

\begin{abstract}
The reorganisation and new circumscription of the gigantic genus Psychotria started with the classic article of Nepokroeff and co-authors in 1999, based on molecular phylogenetic studies. Its statements and suggestions were followed by the separations and modifications of different genera, as the extension of Margaritopsis by Andersson in 2001 and C. M. Taylor in 2005, revalidation and extension of Notopleura by C. M. Taylor in 2001, the new circumscription of the genus Psychotria by Andersson, in 2002, and revalidation of Carapichea by C. M. Taylor in 2012. The major modification of the neotropical Psychotria was the union of the Palicourea and the Heteropsychotria subgenus of Psychotria (Steyermark 1972) suggested by Nepokroeff and co-authors mentioning that this change would provoke the transfer of about 250 neotropical Psychotria species. This process started with the article of C. M. Taylor and co-authors in 2010, followed by Borhidi in 2011 and further changes are forecast by C. M. Taylor in the Flora Mesoamericana (2012). The mentioned modification concerning mainly the species of the Mexican and Mesoamerican flora and some species of wider neotropical distribution. In the Antillean floras Palicourea and Psychotria are treated according the traditional concept of Alain (Cuba in 1962, Hispaniola in 1995) and the monographs of C. M. Taylor in 1988, and 1989. In the recent paper the authors publish the first results of the taxonomic revision of the Psychotria s. 1. species of the Cuban and Hispaniolan flora proposing 4 new combinations and a short actualised review of the taxa concerned the former corrections listed above.
\end{abstract}

Key words: Cuba, nomenclature, Psychotria, taxonomy

\section{INTRODUCCIÓN}

El género Psychotria s. 1. es pantropical y uno de los más grandes entre las Angiospermas contando con 1000 a 1650 especies (Hamilton 1989, Taylor 1996) en los distintos trópicos. Además este es el género más grande de la 
família Rubiaceae, perteneciendo a la subfamília Rubioideae y a la tribu Psychotrieae. El género ha sido considerado como un modelo para el estudio de la filogénia de los mecanismos reproductórios teniendo gran escala del sistema de reproducción, como flores hermafroditas, dístilas y funcionalmente unisexuales. Sin embargo, Psychotria resultó una unidad compleja en sentido taxonómico. Esta complejidad fue revelada por primera vez por el estudio de Nepokroeff et al. (1999) con métodos moleculares y publicaron en seguida una serie de proposiciones para la reorganización taxonómica de este género. Este proceso ha sido seguido por una serie de estudios importantes: La extensión del género Margaritopsis por Andersson (2001), quien encontró que este género descrito originalmente como endémico de Cuba y La Española es un género de distribución pantropical. Su monográfica fue realizada por C. M. Taylor en 2005. Otro paso importante fue la división y revalidación del género Notopleura Bremekamp por C. M. Taylor (2001) la nueva circunscripción del género Psychotria (Andersson 2002a), la revalidación del género Carapichea (Andersson 2002b) y su tratamiento monográfico (Taylor y Gereau 2013). La mayor modificación propuesta por el estudio de Nepokroeff et al. fue la unión del subgénero Heteropsychotria Steyermark del género Psychotria con el género Palicourea. La realización de esta proposición hizó necesario la transferencia de aproximadamente 250 especies de Psychotria a Palicourea. El tamaño de esta operación hizó precautivos los especialistas. Taylor propusó crear un género nuevo para el complejo Palicourea y Heteropsychotria grupo bajo el nombre Psychotrophum, pero la idea no era respaldada. La prioridad del nombre genérico de Palicourea ha sido guardada pero la emendación del nombre y la circunscripción falta todavía. La separación del subgénero Heteropsychotria de la Psychotria s. str. empezó, cuando un grupo de las especies nocturnas Mesoamericanas fue transferido a Palicourea (Taylor et al. 2010) y se continuó con la transferencia de las espécies de las Rubiáceas Mexicanas (Borhidi 2011, 2012). En la Flora Mesoamericana C. M. Taylor trata el subgénero Heteropsychotria, como una unidad separada.

\section{DISCUSSIÓN}

En las Floras de las Antillas (Cuba: Alain 1962; Jamaica: Adams, 1972; La Española: Alain Liogier 1996; Puerto Rico: Alain y Martorell 1982; Antillas Menores: Howard 1989) el subgénero no esta tratada separadamente dentro de Psychotria. Las monografías de C. M. Taylor incluyen las especies de Palicourea en el sentido clásico. Vale la pena mencionar, que algunas especies del Heteropsychotria ya fueron reconocidas y descritas como pertenecientes al género Palicourea en los años de 20 y 30 por Urban $(1922,1923)$ y Standley (1936). Algunos de estos nombres tempranos revalidamos ahora, cuando tienen prio- 
ridad. Tenemos que acentuar, que los estudios recientes de las especies paleotropicales fortalecieron la distancia genética existente entre la Palicourea s. 1. y la Psychotria s. str. separando la Palicorea s. 1. con los géneros estrechamente relacionados (p.e., Margaritopsis, Notopleura, etc.) de la tribu Psychotrieae como una tribu hermana, "sister tribe" autónoma bajo el nombre Palicoureeae (Razafimandimbison et al. 2014).

Después de haber estudiado las especies del género Psychotria s. 1. existentes en la Flora de Cuba, La Española y Jamaica, proponemos las correcciones y/o modificaciones, que corresponden, incluido algunos cambios nomenclatóricos, 1 introducción de nombre nuevo y 8 combinaciones nuevas. Estos son los siguientes.

Margaritopsis Wr. in Sauv. emend. Andersson (2001)

Margaritopsis agustinae (Acuña) C. M. Taylor, Syst. Geogr. Pl. 75: 168 (2005). - Bas.: Psychotria agustinae Acuña, Brittonia 14: 225 (1962).

Margaritopsis microdon (DC.) C. M. Taylor, Syst. Geogr. Pl. 75: 169 (2005). - Bas.: Rondeletia microdon DC., Prodr. 4: 48 (1830). $\equiv$ Psychotria microdon (DC.) Urb., Symb. Ant. 9: 539 (1928). - Tipo: Cuba, Havanna, de La Ossa s.n. Holotipo: G-DC!, microfiche IDC 800.691: III.7, 8. - Syn.: Psychotria pinularis Sessé et Moç., Fl. Mexic., 2. ed., p. 57 (1894).

Margaritopsis nutans (Sw.) C. M. Taylor, Syst. Geogr. Pl. 75: 169 (2005). - Bas.: Psychotria nutans Sw., Prodr. Veg. Ind. Occ., p. 43 (1788). - Tipo: Hispaniola, sin fecha y localidad. Holotipo: Swartz, s.n. S!. - Syn.: Psychotria lineata Humb. et Bonpl. ex Roem et Schult. Syst. 5: 191 (1819). = Psychotria oligantha DC., Prodr. 4: 514 (1830).

Notopleura (Benth.) Bremek. emend. C. M. Taylor (2001)

Notopleura discolor (Griseb.) C. M. Taylor, Ann. Mo. Bot. Gard. 88: 495 (2001). - Bas.: Psychotria uliginosa var. discolor Griseb., Fl. Brit. W. I., p. 340 (1861). - Syn.: Psychotria discolor (Griseb.) Rolfe, Bull. Misc. Inform. Kew 1893: 258 (1893).

Notopleura guadalupensis (DC.) C. M. Taylor, Ann. Mo. Bot. Gard. 88: 512 (2001). - Bas.: Loranthus guadalupensis DC., Prodr. 4: 294 (1830) [” Psychotria guadalupensis (DC.) Howard, J. Arn. Arb. 47: 139 (1967)]. - Tipo: Guadeloupe: Bertero s.n. Holotipo: G-DC!, microfiche: IDC 800.666: II.2. - Syn.: Viscoides pendulum Jacq., Select. Amer. 73, pl. 51, fig. 1 (1763) [三 Psychotria pendula (Jacq.) Urb., Symb. Ant. I: 445 (1900), no Hooker, Fl. Brit. Ind. 3: 164 (1880)]; = Psychotria parasitica Sw., Prodr., p. 44 (1788), nom. illeg. [” Mapouria parasitica (Sw.) K. Schum. in Engler, Nat. Pflanzenfam. 4: 112 (1891); 三 Uragoga parasitica 
(Sw.) Gómez, Anal. Soc. Esp. Nat. II. 3: 293 (1894)]; = Loranthus portoricensis DC., Prodr. 4: 293 (1830), non Psychotria portoricensis DC., Prodr. 4: 515 (1830).

Notopleura guadalupensis subsp. tetrapyrena (Urb.) C. M. Taylor, Ann. Mo. Bot. Gard. 88: 513 (2001). - Bas: Psychotria pendula (Jacq.) Urb. subsp. tetrapyrena Urb., Symb. Ant. 1: 445 (1900). - Tipo: Cuba, Pinal de Santa Ana, 800 m, Wright 249. Holotipo: GOET!, no B, cómo esta citado por C. M. Taylor. - Syn.: Psychotria guadalupensis (DC.) Howard subsp. tetrapyrena Steyerm., Mem. N. Y. Bot. Gard. 23: 570 (1972).

Notopleura uliginosa (Sw.) Bremek., Rec. Trav. Bot. Neerl. 31: 289 (1934). - Bas.: Psychotria uliginosa Sw., Prodr. Veg. Ind. Occ., p. 43 (1788) [三 Uragoga uliginosa (Sw.) Kuntze, Rev. Gen. Pl. 2: 963]. - Tipo: Jamaica, Swartz s.n. Holotipo: S!. - Syn.: Psychotria laevis DC., Prodr. 4: 505 (1830); = Psychotria phytolacca Spreng. ex DC., Prodr. 4: 505 (1830).

\section{Palicourea Aubl. (1775)}

Palicourea acuminata (Benth.) Borhidi, Acta Bot. Hung. 53: 243 (2011). - Bas.: Psychotria acuminata Benth., Bot. Voy. Sulphur, p. 107 (1844). - Tipo: Colombia, Isle de Gorgona, and Colombian coast, Barclay s.n. Holotipo: BM!. - Syn.: Palicourea didymocarpa Griseb., Cat. Pl. Cub., p. 138 (1866) [三 Ronabaea didymocarpa A. Rich. in Sagra, Hist. Fis. Pol. Nat. Cub. 11: 00 (1850); 三Uragoga didymocarpa (A. Rich. ex DC.) Gómez-Maza, Anales Soc. Esp. Hist. Nat. 23: 294 (1894)]; = Psychotria cuspidata Acuña et Roig in Alain H. Liogier Flora de Cuba 5: 105, non Bredem. ex Schult. in Roem. et Schult. Syst. Veg. 5: 192 (1819).

Palicourea agnata (DC.) Borhidi, comb. nova, Acta Bot. Hung. 57: 274 (2015). - Bas.: Psychotria agnata DC., Prodr. 4: 505 (1830). - Tipo: Hispaniola, probablemente en Haiti, colector desconocido. Holotipo: G-DC!.

Palicourea berteroana (DC.) Borhidi, Acta Bot. Hung. 53: 243 (2011). Bas.: Psychotria berteroana DC., Prodr. 4: 515 (1830). - Tipo: Santo Domingo, Dominique, sin localidad precisa, Bertero s.n. Holotipo: G-DC!. - Syn.: Psychotria crebrinervia Standl., Publ. Field Mus. Nat. Hist. Bot. Ser. 4: 343 (1929); = Psychotria platyphylla DC., Prodr. 4: 517 (1830); = Psychotria pyramidalis Griseb., Mem. Amer. Acad., N. S. 8: 509 (1863); = Psychotria hebecladoides Griseb., Mem. Amer. Acad., N. S. 8: 509 (1863).

Palicourea brachiata (Sw.) Borhidi, Acta Bot. Hung. 53: 243 (2011). Bas.: Psychotria brachiata Sw., Prodr. Veg. Ind. Occ., p. 44 (1788). - Tipo: Jamaica: Swartz s.n. Holotipo: S!.

Palicourea corymbosa (Sw.) Borhidi, comb. nova, Acta Bot. Hung. 57: 275 (2015). - Bas.: Psychotria corymbosa Sw., Prodr. Veg. Ind. Occ., p. 44 (1788). - Tipo: Jamaica. Holotipo: S!. 
Palicourea deflexa (DC.) Borhidi subsp. cubensis (Steyerm.) Borhidi et Oviedo comb. nova, Acta Bot. Hung. 57: 275 (2015). - Bas.: Psychotria deflexa DC. subsp. cubensis Steyerm., Mem. NY. Bot. Gard. 23: 505 (1972). - Syn.: Palicourea brachystigma Urb., Ark. Bot. 17(7): 58 (1921). = Psychotria patens Acuña et Roig in Alain, Flora de Cuba 5: 105 (1962). non Sw. 1788. = Psychotria cubensis (Steyerm.) Borhidi Acta Bot. Hung. 37: 86 (1992).

Palicourea dolichantha (Urb.) Borhidi, comb. nova, Acta Bot. Hung. 57: 275 (2015). - Bas.: Psychotria dolichantha Urb., Symb. Ant. 5: 517 (1908). - Tipo: Jamaica. Holotipo: BM.

Palicourea domatiata (C. D. Adams) Borhidi, comb. nova, Acta Bot. Hung. 57: 275 (2015). - Bas.: Psychotria domatiata C. D. Adams, Phytologia 21: 69 (1971). Tipo: Jamaica. Holotipo: BM!.

Palicourea domingensis (Jacq.) DC., Prodr. 4: 529 (1830). - Bas.: Psychotria domingensis Jacq., Enum. Pl. Carib., p. 16 (1760). - Tipo: Santo Domingo: Jacquin, no localizado. - Syn.: Pavetta pentandra Sw., Fl. Ind. Occ. 1: 233 (1788); = Psychotria pavetta Sw., Prodr. Ind. Veg., p. 45 (1797); Palicourea tabernaefolia DC., Prodr. 4: 525 (1830); = Psychotria westii DC., 4: 516 (1830). Tipo: Porto Rico; = Psychotria mombachensis Standl., Publ. Field Mus. Nat. Hist., Bot. Ser. 8: 188 (1930).

Palicourea hoffmannseggiana (Willd. ex Schult.) Borhidi, Acta Bot. Hung. 53: 245 (2011). - Bas.: Cephaëlis hoffmannseggiana Willd. ex Roem. et Schult., Syst. Veg. 5: 214 (1819). - Tipo: Brasil: Pará, coll.: Sieber in Hoffmannsegg, s.n., location unknown (Lorence 1999). - Syn.: Psychotria tribracteata Wr. ex Griseb., Cat. Pl. Cub., p. 137 (1866); = Psychotria hoffmannseggiana subsp. tribracteata (Wr. ex Griseb.) Borhidi, Acta Bot. Hung. 51: 278 (2009).

Palicourea liogieri (Steyerm.) Borhidi, comb. nova, Acta Bot. Hung. 57: 275 (2015). - Bas.: Psychotria liogieri Steyerm., Mem. N. Y. Bot. Gard. 23: 700 (1972). - Tipo: Haiti; in Fonds Verettes, in fruticeto cr. 1000 m., fl. Aug. Buch no. 1236. - Syn.: Uragoga domingensis Urb., Repert. Spec. Nov. Regni Veg. 17: 7 (1921); = Uragoga domingensis var. barahonensis Urb., Spec. Nov. Regni Veg. 17: 8 (1921); = Uragoga domingensis var. fuertesii Urb., Spec. Nov. Regni Veg. 17: 8 (1921); = Cephaëlis domingensis (Urb.) Standl., Publ. Field Mus. Nat. Hist. Bot. Ser. 4: 335 (1929); = Cephälis domingensis var. fuertesii (Urb.) Standl., Publ. Field Mus. Nat. Hist. Bot. Ser. 4: 335 (1929).

Palicourea micrantha Urb. et Ekm., Ark. Bot. 22A (10): 97 (1929). - Tipo: Hispaniola, Santo Domingo; Cordillera Central, in prov. Azua prope Las Lagunas in silvis solo lateritico $750 \mathrm{~m}$. alt., m. Jun. flor., leg.: Ekman H 6410. Holotipo: S!. - Syn.: Psychotria azuensis Alain, Moscosoa 8: 10 (1994), nom. superfl.

Palicourea moensis (Britt. et Wils.) Borhidi, Acta Bot. Hung. 51: 276 (2009). - Bas.: Psychotria moensis Britt. et Wils., Mem. Torrey Bot. Cl. 16: 113 
(1920). - Tipo: Cuba, Oriente, Sierra de Moa, Campo La Gloria, Shafer 8273. Holotipo: NY, isotipo: HAC, US.

Palicourea moralesii (Acuña et Roig) Borhidi, Acta Bot. Hung. 51: 276 (2009). - Bas.: Psychotria moralesii Acuña et Roig, Brittonia 14: 224 (1962). Tipo: Cuba.

Palicourea orientensis Borhidi et Oviedo, nomen novum, Acta Bot. Hung. 57: 276 (2015). - Nombre original: Psychotria subalata Wr. ex Griseb., Cat. Pl. Cub., p. 137 (1866). - Tipo: Cuba oriental, Mayari, Ch. Wright 2754. Holotipo: GOET, isotipo: GH, HAC!.

La utilización de la combinación Palicourea subalata Borhidi et Oviedo sería ilegítimo por la existencia del homónimo válido, que es la Palicourea subalata Standl. ex Steyerm., Acta Biol. Venez. 4: 81 (1964).

Palicourea patens (Sw.) Urb., Repert. Spec. Nov. Regni Veg. 18: 197 (1922). - Bas.: Psychotria patens Sw., Prodr. Veg. Ind. Occ., p. 45 (1788). - Type: Jamaica. Holotype: S!.

Palicourea pedunculata (Sw.) Borhidi, comb. nova, Acta Bot. Hung. 57: 276 (2015). - Bas.: Psychotria pedunculata Sw., Prodr. Veg. Ind. Occ., p. 44 (1788). - Type Jamaica. Holotype: S.

Palicourea polymorpha (Greuter) Borhidi et Oviedo, comb nova, Acta Bot. Hung. 57: 276 (2015). - Bas.: Psychotria polymorpha Greuter, Bissea 3 (num. esp.): 93 (2009). - Tipo: Cuba, Prov. Las Villas, Sierra de Escambray, Pico Potrerillo, 16. Jul 1957, Alain 6398. Holotipo: LS/HAC. - Syn.: Psychotria martii Acuña et Roig., Brittonia 14: 224 (1962), nomen illeg., non Psychotria martii Colla, Herb. Pedem. 3: 141 (1835). Palicourea polymorpha Govaerts, Smithsonian Contr. Bot. 98: 833. 2012, nom illeg.

Palicourea pubescens (Sw.) Borhidi, Acta Bot. Hung. 53: 246 (2011). Bas.: Psychotria pubescens Sw., Prodr. Veg. Ind. Occ., p. 44 (1788). - Tipo: Jamaica, Swartz s.n. Holotipo: S!. - Syn.: Psychotria mollis Poir. in Lam., Encycl. Méth. Bot. 5: 701 (1804); = Psychotria scabriuscula Bartl. ex DC., Prodr. 4: 513 (1830); = Psychotria justicioides Schltdl., Linnaea 9: 596 (1834); = Psychotria glauca Polak., Linnaea 41: 569 (1877).

Palicourea richardiana (Urb.) Borhidi et Oviedo, comb. nova, Acta Bot. Hung. 57: 276 (2015). - Bas.: Psychotria richardiana Urb., Symb. Ant. 7: 472 (1913). - Tipo: Cuba, Prov. Pinar del Rio, Vuelta de Abajo, Valenzuela, s.n. Holotipo: P!. - Syn.: Psychotria involucrata A. Rich. in Sagra, Hist. Fis. Pol. Nat. Cub. 11: 27 (1850), non Sw. (1788); = Palicourea cyanea Urb., Symb. Ant. 9: 168 (1923).

Palicourea toensis (Britt. et Wils.) Standl., Publ. Field Mus. Nat. Hist. Bot. ser. 11: 231 (1936). - Bas.: Psychotria toensis Britt. et Wils., Mem. Torrey Bot. Cl. 16: 113 (1920). - Tipo: Cuba, Prov. Oriente, Campo Toa, Rio Yamani- 
güey, Shafer 4009. Holotipo: NY, isotipo: US. - Syn.: Palicourea purpurascens Urb., Symb. Ant. 9: 167 (1923).

Palicourea torbeciana Urb. et Ekm., Ark. Bot. 21A(5): 87 (1927) [झ Psychotria torbeciana (Ekm. et Urb.) Alain, Moscosoa 8: 10 (1994)]. - Tipo: Hispaniola, Haiti; Massif de la Hotte, in parte occidentali prope Torbec in montibus saxosis supra La Mare Proux, cr. 1200 m. alt., fruct. Dec. leg.: Ekman no. H 5270. Holotipo: S!.

Palicourea violacea (Aubl.) A. Rich., Mem. Rubiac., p. 95 (1829). - Bas.: Nonatelia violacea Aubl., Hist. Pl. Guiane, p. 188 (1775). - Tipo: French Guyana. Holotipo: P!. - Syn.: Psychotria amplifolia Raeusch., Nomencl. Bot., p. 56 (1797).

\section{LITERATURA CONSULTADA}

Acevedo-Rodriguez, P. and Strong, M.T. (2012): Catalogue of the seed plants of West Indies. - Smithsonian Contr. Bot. 98: 1-1192.

Adams, D. C. y Proctor, G. R. (1972): Rubiaceae. - In: Flowering Plants of Jamaica. Univ. W. I., Mona, Jamaica, pp. 699-734.

Alain, H. Liogier (1962): Rubiaceae, Flora de Cuba, Vol. V. - Univ. Porto Rico, Rio Piedras, pp. 13-148.

Alain, H. Liogier (1995): Rubiaceae, Flora de la Española. Vol. VII. - San Pedro de Macorís, R. D., pp. 207-439.

Andersson, L. (2001): Margaritopsis (Rubiaceae, Psychotrieae) is a pantropical genus. Syst. Geogr. Pl. 71: 73-85.

Andersson, L. (2002a): Relationships and generic circumscriptions in the Psychotriacomplex (Rubiaceae, Psychotrieae). - Syst. Geogr. Pl. 72: 167-202. http://dx.doi. org/10.2307/3668767

Andersson, L. (2002b): Re-establishment of Carapichea (Rubiaceae, Psychotrieae). - Kew Bull. 57: 363-374. http://dx.doi.org/10.2307/4111112

Andersson, L. y Rova, J. H. E. (1999): The rps16 intron and the phylogeny of the Rubiaceae. - Plant Syst. Evol. 214: 161-186. http://dx.doi.org/10.1007/BF00985737

Borhidi, A. (2011): Transfer of the Mexican species of Psychotria subgen. Heteropsychotria to Palicourea based on morphological and molecular evidences. - Acta Bot. Hung. 53: 241-250. http://dx.doi.org/10.1556/ABot.53.2011.3-4.4

Borhidi, A. (2012): Rubiáceas de México. Segunda y ampliada edición. - Akadémiai Kiadó, Budapest, 610 pp.

Borhidi, A., Fernández-Zequeira, M. y Oviedo-Prieto, R. (2009): Species combinationesque nonnullae novae Florae Cubanae. - Acta Bot. Hung. 51: 273-282. http://dx.doi. org/10.1556/ABot.51.2009.3-4.4

Govaerts, R. (2014): World checklist of Rubiaceae. - Royal Botanic Gardens, Kew. http://apps. kew.org/wcsp

Grisebach, A. R. (1866): Catalogus Plantarum Cubensium. - Lipsiae.

Hamilton, C. W. (1989): A revision of Mesoamerican Psychotria subgenus Psychotria (Rubiaceae). - Ann. Mo. Bot. Gard. 76: 67-111, 386-429, 886-916. http://dx.doi. org/10.2307/2399343

Howard, R. A. (1979): Flora of the West Indies. - In: Larsen, K. (ed.): Tropical botany. Academic Press, London, pp. 239-250. 
Howard, R. A. (1988): Charles Wright in Cuba, 1856-1867. - Chadwyck-Healey, Cambridge, England, $90 \mathrm{pp}$.

Howard, R. A. (1989): Rubiaceae. - Flora of the Lesser Antilles 6: 389-471.

Nepokroeff, M., Bremer, B. y Sytsma, K. J. (1999): Reorganization of the genus Psychotria and tribe Psychotrieae (Rubiaceae) inferred from ITS and rbcL sequence data. - Syst. Bot. 24(1): 5-27. http://dx.doi.org/10.2307/2419383

Razafimandimbison, S. G., Taylor, C. M., Wikström, N., Pailler, T., Khodabandeh, A. y Bremer, B. (2014): Phylogeny and generic limits in the sister tribes Psychotrieae and Palicoureeae (Rubiaceae): evolution of schizocarps in Psychotria and origin of bacterial leaf-nodules of the Malgasy species. - Amer. J. Bot. 101(7): 1102-1126. http:// dx.doi.org/10.3732/ajb.1400076

Richard, A. (1850): Rubiaceae. - In: de la Sagra, R.: Historia Física Política y Natural de la Isla de Cuba. XI. Paris, pp. 3-28.

Steyermark, J. A. (1972): Rubiaceae. In: Maguire, B., Wurdack, J. J. et al. (eds): Botany of the Guayana Highlands. Part IX. - Mem. N. Y. Bot. Gard. 23: 227-832.

Steyermark, J. A. (1974): Rubiaceae. - In: Lasser, T. y Steyermark, J. A. (eds): Flora de Venezuela 9(1-3): 1-2070. Caracas, Inst. Bot.

Taylor, C. M. (1987): Reconsideration of the generic placement of Palicourea domingensis (Rubiaceae, Psychotrieae). - Ann. Mo. Bot. Gard. 74: 447-448. http://dx.doi. org $/ 10.2307 / 2399421$

Taylor, C. M. (1989): Revision of Palicourea in Mexico and Central America. - Syst. Bot. Monogr. 26: 1-102. http://dx.doi.org/10.2307/25027720

Taylor, C. M. (1993): Revision of Palicourea (Rubiaceae: Psychotrieae) in the West Indies. Moscosoa 7: 201-241.

Taylor, C. M. (1996): Overview of the Psychotrieae (Rubiaceae) in the Neotropics. - Opera Bot. Belg. 7: 261-270.

Taylor, C. M. (2001a): Rubiaceae Juss. In: Stewens, W. D. et al. (eds): Flora de Nicaragua. Ann. Mo. Bot. Gard. 85: 2206-2284.

Taylor, C. M. (2001b): Overview of the neotropical genus Notopleura (Rubiaceae: Psychotrieae), with description of some new species. - Ann. Mo. Bot. Gard. 88: 478-515.

Taylor, C. M. (2004): Rubiacearum Americanarum Magna Hama XVI. New species, a new subspecies, and an overlooked species of Psychotria subg. Heteropsychotria from Mexico, Central America, and Western South America. - Novon 14(4): 193-208.

Taylor, C. M. (2005): Margaritopsis (Rubiaceae, Psychotrieae) in the Neotropics. - Syst. Geogr. Pl. 75(2): 161-177.

Taylor, C. M. y Gereau, R. T. (2013): The genus Carapichea (Rubiaceae, Psychotrieae). Ann. Mo. Bot. Gard. 99: 100-127. http://dx.doi.org/10.3417/2011064

Urban, I. (1913): Ad cognitionem generis Psychotriae additamenta. - Symb. Antillanae 7: 435-477. 